\title{
Occurrence of Urinary Tract Infection in Patients with Renal Allograft Biopsies Showing Neutrophilic Tubulitis
}

Luciano Espinheira Fonseca, Jr., M.D., Ron Shapiro, M.D., Parmjeet S. Randhawa, M.D. Department of Anatomic Pathology \& Legal Medicine (LEF), Bahia Federal Medical School, Salvador, Bahia, Brazil; and Departments of Surgery (RS) and Pathology (PSR), University of Pittsburgh School of Medicine, Pittsburgh, Pennsylvania

Lymphocytic tubulitis is a well-accepted criterion for acute cellular rejection in renal allograft biopsies. Neutrophilic tubulitis has been used as a surrogate marker for urinary tract infection, but it is not clear how reliably this lesion can be used to make this diagnosis. Biopsy findings were correlated with clinical features in 26 renal allograft biopsies with interstitial polymorphonuclear infiltrates associated with neutrophilic tubulitis. The grade of neutrophilic tubulitis exceeded the grade of lymphocytic tubulitis in 7 (44\%) of 16 patients with, but in only 0 patients without, a positive urine culture. Culture confirmed urinary tract infection in 16 $(62 \%)$ of 26 patients. It is possible that prior antibiotic therapy led to a false-negative culture and masked the diagnosis in two additional patients. Lymphocytic tubulitis made it difficult to exclude concurrent acute cellular rejection in all biopsies studied. In 6 (23\%) of 26 patients, negative cultures and response to steroid treatment confirmed that neutrophilic tubulitis can occur in biopsies without urinary tract infection. The relative contributions of infection and rejection could not be determined in patients treated with both steroids and antibiotics. Neutrophilic tubulitis in a renal allograft biopsy should alert the clinician to the possibility of urinary tract infection, even if concurrent lymphocytic tubulitis is present. Confirmation by urine culture is needed because biopsies with ischemic injury and acute cellular or antibody-mediated rejection can show overlapping histology.

Copyright () 2003 by The United States and Canadian Academy of Pathology, Inc.

VOL. 16, NO. 4, P. 281, 2003 Printed in the U.S.A

Date of acceptance: January 28, 2003.

This work was supported by The Thomas E Starzl Transplantation Institute, University of Pittsburgh, Pittsburgh, PA.

Address reprint requests to: Parmjeet S. Randhawa, M.D., Division of Transplantation Pathology, Department of Pathology, C903.1 Presbyterian University Hospital, University of Pittsburgh, Pittsburgh, PA 15213; fax: 412-647-5237; e-mail: randhawapa@msx.upmc.edu.

DOI: 10.1097/01.MP.0000062658.47481.69
KEY WORDS: Allograft, Kidney, Renal, Transplantation, Urinary tract infection.

Mod Pathol 2003;16(4):281-285

Kidney transplantation is now a well-accepted treatment modality for end-stage kidney disease. When graft dysfunction occurs, the renal biopsy plays an indispensable role in defining the underlying cause and in assisting the clinician in determining the most suitable therapeutic intervention (1-3). Lymphocytic tubulitis, defined as the presence of lymphocytes on the internal aspect of the tubular basement membranes, is a welldocumented criterion for the diagnosis of acute cellular rejection. The significance of neutrophilic tubulitis has been less extensively studied. Standard pathology textbooks do mention the presence of neutrophil interstitial infiltrates in the setting of urinary tract infection $(4,5)$. However, the actual frequency with which polymorphonuclear cells in an allograft biopsy are associated with culture-proven infection is not defined. It is also uncertain whether the concurrent presence of mononuclear infiltrates and lymphocytic tubulitis in these biopsies warrants an alternate or additional diagnosis of acute cellular rejection. This study has been designed to address these issues and is based on clinicopathologic correlations performed on 26 renal allograft biopsies, wherein initial histologic examination raised the possibility of urinary tract infection.

\section{MATERIALS AND METHODS}

Patients were selected from a database maintained by The Thomas E. Starzl Transplantation Institute at the University of Pittsburgh. This database contains biopsies coded into specific diagnostic categories by one of the authors (PR). For the purposes of this study, we retrieved 26 biopsies performed for rising serum creatinine and coded as interstitial nephritis with neutrophils. This diagnostic entity encompasses specimens showing intersti- 
tial inflammation, focal or diffuse, associated with neutrophilic tubulitis, defined as neutrophil infiltration in the tubular epithelium. Cases in which the neutrophils were associated with a positive antibody cross-match, or viral inclusions in the biopsy tissue, were not included in this investigation. The samples studied constitute approximately $3 \%$ of all renal allograft biopsies accessioned in our department over a period of 4 years.

Histopathologic examination of these specimens was performed using The Banff Schema of Renal Allograft Pathology $(6,7)$. The intensity of interstitial inflammation was graded from i0 to i3, depending on the percentage of biopsy area involved. Lymphocytic tubulitis was defined as the presence of mononuclear cells on the internal aspect of the tubular basement membranes and graded from t0 through t3. Neutrophilic tubulitis was separately graded using the same criteria. Chronic allograft nephropathy was recognized and graded using a semiquantitative evaluation of the biopsy for interstitial fibrosis, tubular atrophy, arteriosclerosis, and duplication of glomerular basement membranes. Pertinent clinical information was obtained by review of medical records. Serum creatinine at the time of clinical presentation was used as an index of renal allograft dysfunction. Results of anti-rejection treatment were characterized as (1) complete response if the serum creatinine fell by $\geq 70 \%$ of the initial rise, (2) no response if the drop was $<30 \%$, and (3) partial response if cases fell between the aforementioned extremes. Urine cultures were con- sidered clinically significant if $>100,000$ organisms per $\mathrm{mL}$ were isolated. The data collection protocol used in this study was approved by The University of Pittsburgh Institutional Review Board (IRB Protocol 020133).

\section{RESULTS}

The demographic characteristics of the subjects studied are typical of patients accrued into our transplant program (Table 1). There were 13 males and 13 females varying in age from 23 to 77 years. The causes of end-stage kidney disease culminating in transplantation were diabetes mellitus $(n=3)$, glomerulonephritis $(n=4)$, systemic lupus erythematosus $(n=3)$, hypertension $(n=5)$, reflux nephropathy (1), pyelonephritis $(n=2)$, and miscellaneous $(n=8)$. The biopsies examined had been performed 1-968 weeks (mean, $125 \mathrm{wk}$ ) after transplantation.

On histopathologic examination, all biopsies, by definition, had areas of interstitial inflammation with neutrophil predominance (Fig. 1). Polymorphonuclear cells were present infiltrating the tubular epithelium (neutrophilic tubulitis) and also formed small clusters in the tubular lumen. Interstitial polymorphonuclear cells and neutrophilic tubulitis were generally seen in close proximity to each other. Neutrophilic casts were recognized in 10 biopsies (Fig. 2). The grade of neutrophilic tubulitis exceeded the grade of lymphocytic tubulitis in

TABLE 1. Clinical and Pathologic Features of Cases Studied

\begin{tabular}{|c|c|c|c|c|c|c|c|c|c|c|c|c|}
\hline Number & $\begin{array}{l}\text { Age } \\
\text { (Yr) }\end{array}$ & Sex & Native Kidney Disease & $\begin{array}{l}\text { Biopsy } \\
\text { Week }\end{array}$ & $\begin{array}{c}\text { Urine } \\
\text { Culture }\end{array}$ & $\begin{array}{c}\text { Lymphocytic } \\
\text { Tubulitis } \\
\text { Score }\end{array}$ & $\begin{array}{c}\text { Neutrophilic } \\
\text { Tubulitis } \\
\text { Score }\end{array}$ & $\begin{array}{l}\text { Inflammation } \\
\text { Score }\end{array}$ & $\begin{array}{l}\text { Neutrophil } \\
\text { Casts }\end{array}$ & $\begin{array}{l}\text { Rejection } \\
\text { Type }\end{array}$ & $\begin{array}{c}\text { Chronic } \\
\text { Allograft } \\
\text { Nephropathy }\end{array}$ & $\begin{array}{l}\text { Creatinine } \\
\text { Response }\end{array}$ \\
\hline 1 & 77 & M & Polycystic kidney & 26 & Positive & 2 & 3 & 2 & No & $1 \mathrm{~A}$ & None & None \\
\hline 2 & 68 & M & Diabetic nephropathy & 49 & Positive & 2 & 2 & 3 & Yes & $1 \mathrm{~A}$ & None & Partial \\
\hline 3 & 49 & $\mathrm{~F}$ & Glomerulonephritis & 3 & Positive & 3 & 3 & 3 & Yes & $1 \mathrm{~B}$ & Moderate & Partial \\
\hline 4 & 23 & $\mathrm{~F}$ & Obstructive uropathy & 181 & Positive & 3 & 3 & 3 & Yes & $1 \mathrm{~B}$ & Severe & None \\
\hline 5 & 40 & $\mathrm{~F}$ & Chronic pyelonephritis & 79 & Positive & 2 & 3 & 2 & No & $1 \mathrm{~A}$ & Moderate & None \\
\hline 6 & 33 & $\mathrm{~F}$ & Lupus nephritis & 136 & Positive & 1 & 3 & 2 & Yes & $1 \mathrm{~A}$ & Moderate & None \\
\hline 7 & 41 & $\mathrm{~F}$ & Lupus nephritis & 200 & Positive & 3 & 3 & 3 & No & $1 \mathrm{~B}$ & Moderate & Complete \\
\hline 8 & 51 & M & Hypertensive nephropathy & 1 & Positive & 2 & 3 & 3 & Yes & $1 \mathrm{~A}$ & None & Complete \\
\hline 9 & 45 & $\mathrm{~F}$ & Pyelonephritis & 968 & Positive & 2 & 2 & 1 & Yes & Borderline & Moderate & Partial \\
\hline 10 & 44 & $\mathrm{~F}$ & Alport syndrome & 427 & Positive & 2 & 3 & 2 & No & Borderline & Mild & None \\
\hline 11 & 31 & M & IgA nephritis & 11 & Positive & 2 & 2 & 2 & Yes & $1 \mathrm{~A}$ & None & None \\
\hline 12 & 74 & M & Hypertensive nephropathy & 13 & Positive & 2 & 3 & 2 & No & $1 \mathrm{~A}$ & None & Partial \\
\hline 13 & 56 & M & Hypertensive nephropathy & 4 & Positive & 3 & 2 & 2 & Yes & $1 \mathrm{~B}$ & None & Complete \\
\hline 14 & 54 & M & Chronic glomerulonephritis & 443 & Positive & 2 & 2 & 2 & No & $1 \mathrm{~A}$ & None & Complete \\
\hline 15 & 33 & $\mathrm{~F}$ & Diabetic nephropathy & 230 & Positive & 3 & 3 & 3 & Yes & $1 \mathrm{~B}$ & Moderate & Complete \\
\hline 16 & 59 & M & Lupus nephritis & 14 & Positive & 1 & 3 & 3 & No & $1 \mathrm{~B}$ & None & Partial \\
\hline 17 & 45 & M & Hypertensive nephropathy & 1 & Negative & 1 & 1 & 1 & No & Borderline & None & Partial \\
\hline 18 & 57 & M & Diabetic nephropathy & 1 & Negative & 3 & 3 & 3 & No & $1 \mathrm{~B}$ & None & Partial \\
\hline 19 & 55 & $\mathrm{~F}$ & Thrombotic angiopathy & 1 & Negative & 3 & 3 & 2 & No & $1 \mathrm{~B}$ & None & Complete \\
\hline 20 & 33 & M & Focal segmental sclerosis & 316 & Negative & 2 & 1 & 2 & No & $1 \mathrm{~A}$ & None & No data \\
\hline 21 & 41 & $\mathrm{~F}$ & IgA nephritis & 1 & Negative & 3 & 3 & 2 & No & $1 \mathrm{~B}$ & None & Complete \\
\hline 22 & 53 & $\mathrm{~F}$ & Focal segmental sclerosis & 10 & Negative & 3 & 3 & 3 & No & $1 \mathrm{~B}$ & None & None \\
\hline 23 & 48 & $\mathrm{~F}$ & Horseshoe kidney & 143 & Negative & 3 & 1 & 3 & No & $1 \mathrm{~B}$ & Moderate & None \\
\hline 24 & 26 & M & Reflux nephropathy & 5 & Negative & 1 & 1 & 1 & No & Borderline & None & None \\
\hline 25 & 40 & $\mathrm{~F}$ & Hypertensive nephropathy & 1 & Negative & 3 & 2 & 3 & Yes & $1 \mathrm{~B}$ & None & Complete \\
\hline 26 & 56 & M & Nephrolithiasis & 2 & Negative & 2 & 1 & 2 & No & $1 \mathrm{~A}$ & None & Complete \\
\hline
\end{tabular}




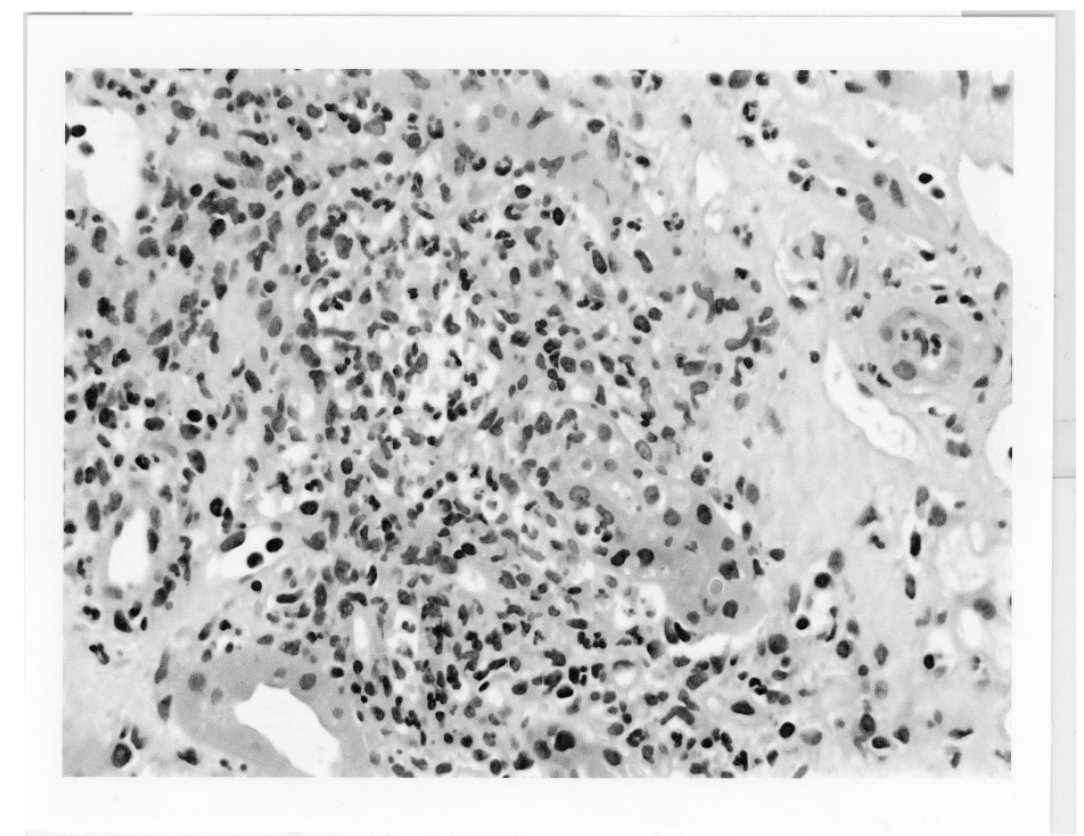

FIGURE 1. A renal allograft biopsy with interstitial nephritis showing features suggestive of an infectious etiology. The interstitial inflammatory infiltrate contains several neutrophils recognizable by their multi-lobate nuclei. Some of these polymorphonuclear cells are seen to infiltrate the tubular epithelium, resulting in neutrophilic tubulitis (H\&E $400 \times)$.

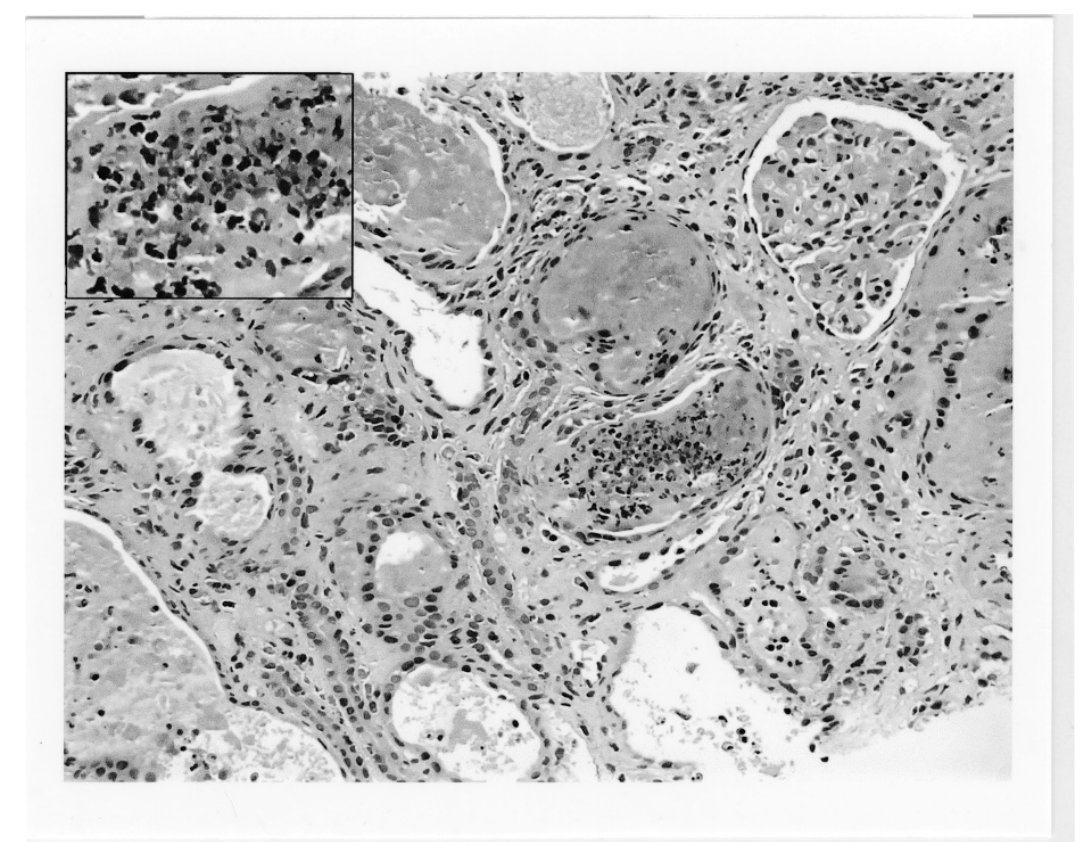

FIGURE 2. Another photomicrograph from the same biopsy as in Figure 1. An atrophic tubule is distended by a proteinaceous cast containing disintegrating inflammatory cells $(\mathrm{H} \& \mathrm{E} ; 200 \times)$. A higher-power insert $(600 \times)$ shows some multilobed nuclear fragments consistent with neutrophils.

7 of $16(44 \%)$ patients with and 0 of $10(0 \%)$ patients without a positive urine culture. In 8 of 10 cases in which this feature could be evaluated, medullary inflammation was more intense than was cortical inflammation. The remaining biopsies sampled only cortical tissue. No viral inclusions, bacteria, or fungal organisms were recognized.

The aforementioned findings were used to suggest the possibility of a urinary tract infection; this possibility was further investigated by sending a urine specimen for microbiologic culture. In all, 18 microorganisms were isolated from 16 patients, with the organisms identified being Enterococcus, Eschericia coli, Klebsiella, Staphylococcus, Streptococcus, and Lactobacillus in four, one, two, five, two, and four isolates, respectively. Two patients had more than one organism cultured from the same urine sample. A course of antibiotics was 
administered to all patients with positive urine culture and to two others (Patients 17 and 18) on clinical grounds before the urine culture was reported as negative. One of the latter two patients had pneumonia but no evidence of urinary tract infection.

In addition to features suggestive of infection noted above, all biopsies had areas of mononuclear interstitial inflammation with tubulitis. The intensity of interstitial inflammation was graded as i1, i2, and i3 in 3, 12, and 11 biopsies, respectively. The scores for lymphocytic tubulitis assigned to these specimens were $\mathrm{t} 1, \mathrm{t} 2$, and $\mathrm{t} 3$ in 4,11 , and 11 instances, respectively. The presence of mononuclear interstitial inflammation and lymphocytic tubulitis in these biopsies made it difficult to exclude concurrent acute cellular rejection. In terms of The Banff Schema, the inflammatory infiltrates and tubulitis in these biopsies corresponded to borderline change in 4 biopsies, Type $1 \mathrm{~A}$ acute rejection in 10 biopsies, and Type 1B acute rejection in 12 biopsies, respectively.

Because of an inability to definitely exclude acute rejection, all patients except Patients 2 and 24 received steroid treatment. The response to antirejection treatment was difficult to assess, because many patients also received a concurrent course of antibiotics. In the eight patients (Patients 19-26) who received only steroids, and no antibiotics, a complete response defined by fall in serum creatinine was observed in four patients. In all seven patients without complete clinical response (Patients 3, 4, 5, $6,9,10$, and 23), biopsy-documented chronic allograft nephropathy was indicated.

\section{DISCUSSION}

In the absence of acute tubular necrosis (8) and antibody-mediated rejection (9), interstitial neutrophils in renal allograft biopsies are used as a surrogate marker for urinary tract infection $(4,5)$. The reliability of this practice has not been specifically investigated. In this study, culture confirmed the presence of urinary tract infection in 16 (62\%) of 26 patients in which it was suspected as a result of biopsy findings. It is possible that prior antibiotic therapy led to a false-negative culture and masked the diagnosis in two additional patients. In 5 of 10 biopsies with a negative culture, biopsy was performed 1 week after transplantation, and the polymorphonuclear cell infiltration was likely the result of ischemic injury to the tubules. Although no staining for the complement component C4d was performed, a negative cross-match makes antibodymediated rejection an unlikely explanation for the neutrophils in these biopsies.

Biopsies taken in the context of clinically proven urinary tract infection frequently showed intersti- tial mononuclear infiltrates and lymphocytic tubulitis. This suggested the concurrent presence of acute cellular rejection in these patients. Clinical response to steroid treatment in several cases was consistent with this impression, although it could be argued that the improvement could, at least in part, have been the result of concurrent antibiotic treatment. Our results are similar to those of Yang et al. (10), who reported acute rejection superimposed on pyelonephritis in $8(25.8 \%)$ of 31 patients studied by them. As to the underlying mechanism, it is likely that intragraft inflammation of infectious etiology results in local release of cytokines such as interferon- $\gamma$ and tumor necrosis factor- $\alpha$ (11), which up-regulate the expression of major histocompatibility antigens and precipitate acute rejection. Another potential mechanism is suggested by Wrishko et al. (12), who report an increased rate of acute cellular rejection in patients receiving ciprofloxacin. This antimicrobial agent antagonizes the action of cyclosporine and is believed to result in an increased concentration of interleukin-2 within the allograft.

In the biopsies with negative cultures, it is likely that influx of neutrophils was a secondary reaction to ischemic and/or immunologic tubular injury. Increased concentrations of interleukin-8, a chemoattractant for neutrophils, have been noted in the urine of patients with acute rejection. A hypersensitivity reaction to drugs, or to a systemic focus of infection, would be a potential alternate explanation for the interstitial nephritis present in these biopsies. However, no incriminating drugs could be identified during a retrospective review of the medical records. Furthermore, no significant eosinophils were present in the inflammatory infiltrates, making at least a Type 1 hypersensitivity reaction unlikely. Interstitial nephritis due to unusual infectious organisms, such as Corynebacterium, Мycoplasma, Chlamydia, and Gardnerella, which evade identification by routine culture, cannot be excluded (13-17).

In conclusion, neutrophilic tubulitis in a renal allograft biopsy should raise the possibility of urinary tract infection, particularly if acute tubular necrosis and antibody-mediated injury can be excluded. Urine cultures are necessary for definitive diagnosis. The presence of concurrent lymphocytic tubulitis is difficult to interpret. In some cases, lymphocytes in the inflammatory infiltrate are probably an epiphenomenon, but in other cases, these might reflect true acute cellular rejection precipitated by infection. A third possibility is that cytokine release in acute cellular rejection without concurrent infection may lead to secondary neutrophil influx. 


\section{REFERENCES}

1. Colvin RB. The renal allograft biopsy. Kidney Int 1996;50: 1069-82.

2. Pardo-Mindan FJ, Salinas-Madrigal L, Idoate M, Garola R, Sola I, French M. Pathology of renal transplantation. Semin Diagn Pathol 1992;9:185-99.

3. Pascual M, Vallhonrat H, Cosimi AB, Tolkoff-Rubin N, Colvin $\mathrm{RB}$, Delmonico FL, et al. The clinical usefulness of the renal allograft biopsy in the cyclosporine era: a prospective study. Transplantation 1999;67:737-41.

4. Colvin R. Bacterial/fungal pyelonephritis. In: Jennette JC, Olson JL, Schwartz MM, Silva FG, editors. Heptinstall's pathology of the kidney. Vol 2. Philadelphia, PA: LippincottRaven; 1998. p. 1496-7.

5. Kashgarian M. Tubulo-interstitial diseases. In: Silva FG, D’Agati VD, Nadasady T, editors. Renal biopsy interpretation. New York: Churchill Livingstone; 1996. p. 309-14.

6. Racusen LC, Solez K, Colvin RB, Bonsib SM, Castro MC, Cavallo T, et al. The Banff 97 working classification of renal allograft pathology. Kidney Int 1999;55:713-23.

7. Solez K, Benediktsson H, Cavallo T, Croker B, Demetris AJ, Drachenberg C, et al. Report of the Third Banff Conference on Allograft Pathology (July 20-24, 1995) on classification and lesion scoring in renal allograft pathology. Transplant Proc 1996;28:441-4.

8. Koo DDH, Welsh KI, Roake JA, Morris PJ, Fuggle SV. Ischemia/reperfusion injury in human kidney transplantation-an immunohistochemical analysis of changes after reperfusion. Am J Pathol 1998;153:557-66.

9. Trpkov K, Campbell P, Pazderka F, Cockfield S, Solez K, Halloran PF. Pathologic features of acute renal allograft rejection associated with donor-specific antibody, analysis using the Banff grading schema. Transplantation 1996;61:1586-92.

10. Yang CW, Lee SH, Choi YJ, Kim YS, Kim SY, Choi EJ, et al. Evaluation of acute renal failure in bacterial allograft pyelonephritis using abdominal CT and graft biopsy. Am J Nephrol 1997;17:42-5.

11. Molvig J, Baek L, Christensen P, Manogue KR, Vlassara H, Platz P, et al. Endotoxin-stimulated human monocyte secretion of interleukin 1, tumour necrosis factor alpha, and prostaglandin E2 shows stable interindividual differences. Scand J Immunol 1988;27:705-16.

12. Wrishko RE, Levine M, Primmett DR, et al. Investigation of a possible interaction between ciprofloxacin and cyclosporine in renal transplant patients. Transplantation 1997; 64:996-9.

13. Birch DF, D'Apice AJ, Fairley KF. Ureaplasma urealyticum in the upper urinary tracts of renal allograft recipients. J Infect Dis 1981;144:123-7.

14. Dimitrakov D, Dimitrakov J, Beleva R. Frequency and clinical characteristics of Mycoplasma urinary tract infections in the early post-transplantation period in renal allograft patients. Folia Med (Plovdiv) 1999;41:59-61.

15. Dimitrakov J, Mourdjeva M, Draganov M, Dimitrakov D. A case of Chlamydia trachomatis infection in a renal allograft patient. Folia Med (Plovdiv) 1998;40:45-7.

16. Hertig A, Duvic C, Chretien Y, Jungers P, Grunfeld JP, Rieu P. Encrusted pyelitis of native kidneys. J Am Soc Nephrol 2000; 11:1138-40.

17. Wanic-Kossowska M, Koziol L, Bajew L, Czekalski S. Acute and chronic urinary tract infections caused by Chlamydia trachomatis. Int Urol Nephrol 2001;32:437-8. 